\title{
Celulitis gangrenosa cervical complicada con mediastinitis. Caso clínico
}

\author{
S. Aboul-hosn Centenero
}

\begin{abstract}
Resumen: Presentamos un caso clínico de celulitis gangrenosa cervical de origen odontógeno que progresó al espacio mediastínico, comentando la importancia de un tratamiento precoz y agresivo (quirúrgico y antibioterapia endovenosa). Discutimos la necesidad de realizar sistemáticamente traqueostomía, así como el momento de llevar a cabo la exodoncia de las piezas causantes del proceso infeccioso.
\end{abstract}

Palabras clave: Celulitis Cervical Odontogénica; Mediastinitis; Traqueostomía.

\begin{abstract}
This is the presentation of a case of cervical necrotizing celullitis which spreads to the mediastinic space, resulting from direct extensión, with an odontogenic origin. Our aims are to explain the importance of a rapid and agressive treatment (surgical and antibiotherapy). We shall discuss the necessity of givin the tracheostomy in all cases, as well as the treatment of extraction of the tooth when is the cause of the infection.
\end{abstract}

Key words: Odontogenic Cervical Celullitis; Mediastinitis; Tracheostomy.

\section{Introducción}

Los procesos infecciosos a nivel del espacio submandibular son una patología frecuente dentro de un Servicio de urgencias de Cirugía Maxilofacial y Oral, no siendo tan común su progresión hacia espacios cervicales contiguos, o hacia espacios mediastínicos. Dentro de estos procesos, una gran mayoría tiene un origen odontógeno,,$^{1,2}$ citándose la patología de las piezas molares inferiores como causa más frecuente. ${ }^{3}$ La agresividad de los gérmenes aerobios y anaerobios intraorales y su sinergismo son los responsables del curso fulminante que en ocasiones sigue esta patología, ${ }^{1,2}$ siendo para muchos autores el Streptococo el germen que más frecuentemente se encuen-

Médico Residente de Cirugía Oral y Maxilofacial

«ospital Universitari de Bellvitge», Barcelona, España

Correspondencia:

S. Aboul-hosn Centenero

Crta. de Arbúcies 4, 17400 Breda, Girona, España.

E-mail: 36002sac@comb.es tra en esta patología7. Entre las múltiples complicaciones está la diseminación directa a través de los diferentes planos anatómicos cervicales; especial mención merece la vía de diseminación para y retroesofágica, hacia mediastino, ${ }^{4}$ con el consiguiente aumento de la morbi-mortalidad pudiendo llegar a ser de hasta un $50 \% .5,6$

\section{Caso clínico}

Varón de 42 años de edad que acude a urgencias por tumefacción y dolor submandibular bilateral de dos días de evolución y que no remitió con tratamiento analgésico y antibiótico oral durante las 48-72 horas previas. Como antecedentes médicos de interés destaca únicamente el hecho de ser fumador de 2 paquetes/día y un enolismo severo. El paciente refiere dolor en piezas molares del cuarto cuadrante de 10-15 días de evolución. A la exploración física destaca un trismus severo junto disnea y odinofagia como signos de gravedad, una boca sépica y una tumefacción submandibular de características inflamatorias más evi- 


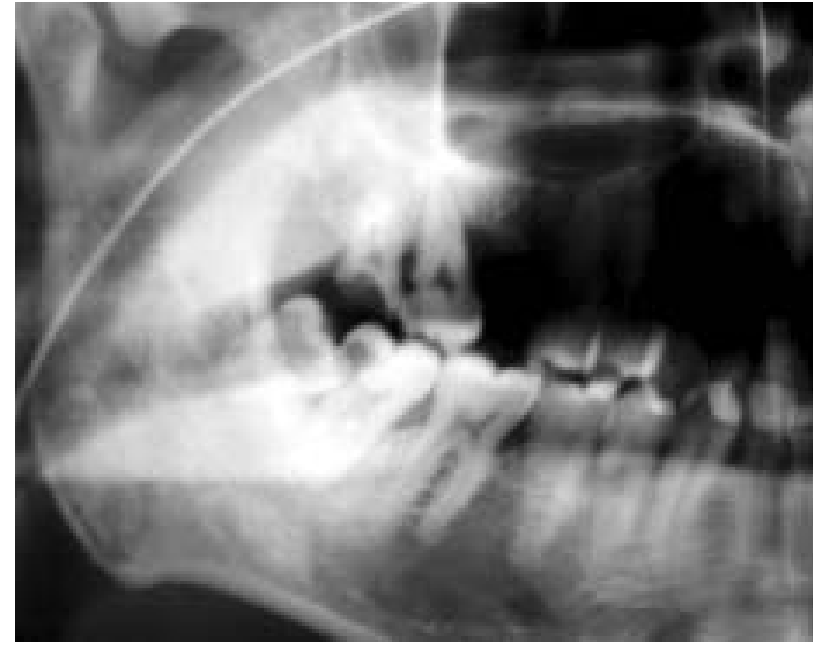

Figura 1. Pieza 48 incluida y mesializada provocando una caries por distal de 47. Pieza 18 presenta necrosis de su corona por caries.

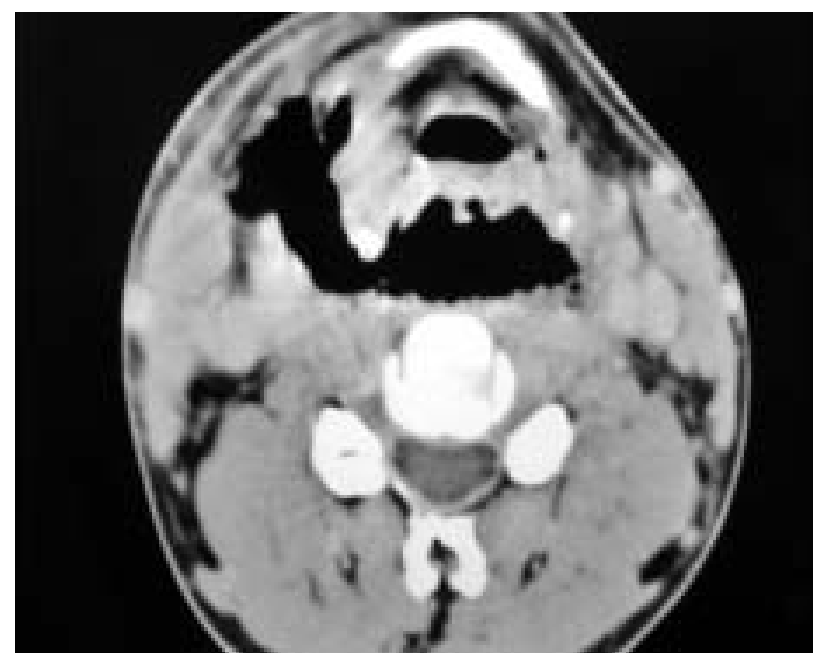

Figura 3. Gran disección de espacios cervicales por acúmulo de gas en región laterocervical derecha que se comunica con gas a nivel retroesofágico.

dente en lado derecho así como crepitación láterocervical ipsilateral. A la exploración intraoral llama la atención una boca séptica con dolor sobre piezas 47 y 48 a la percusión.

En la analítica general, el único dato que llama la atención es una leucocitosis de 15.000x109/l con ligera desviación a la izquierda. La saturación arterial de oxígeno era del 98\%, placa de tórax sin signos patológicos y temperatura axilar de $37,5^{\circ} \mathrm{C}$. En la ortopantomografía se aprecia un 48 mesializado e impactado sobre la pieza 47, que a su vez muestra una caries por distal y caries destructiva de la corona de la pieza 18 (Fig. 1). Se realiza una TC cervical donde se aprecia una gran disección de planos cervicales desde región submandibular hasta últimos cortes cervicales mostrando gran cantidad de densidad gas a nivel laterocervical y retroesofágico (Figs. 2 y 3 ) así como desviación de la vía aérea hacia el lado izquierdo e ima-



Figura 2. Imagen de gas a nivel submandibular, retrofaringeo y laterocervical posterior derecho junto con imagen de hipodensidad compatible con material purulento en cara lingual mandibular derecha.

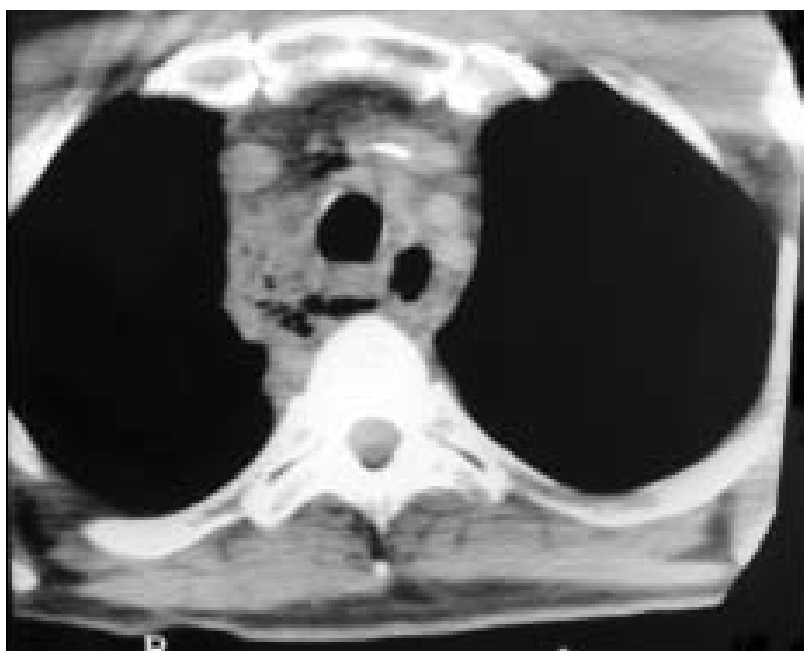

Figura 4. Presencia de densidad gas en el corte axial a nivel del mediastino posterior.

gen compatible con posible material purulento en zona pterigoidea y submandibular derechas.

Realizamos una ampliación de los cortes axiales hasta espacio mediastínico donde se aprecia imagen compatible con colección purulenta y gas a nivel de mediastino posterior (Fig. 4). Se inicia antibioterapia empírica endovenosa con amoxicilina-clavulánico $2 \mathrm{~g}$ $125 \mathrm{mg}$ cada 8 horas y gentamicina $80 \mathrm{mg}$ cada 8 horas.

Junto con el servicio de Cirugía Torácica se interviene de urgencia al paciente practicándose una amplia incisión cervical siguiendo el borde anterior del músculo esternocleidomastoideo y desbridamiento quirúrgico con salida de abundante material necróticopurulento tanto cervical como torácico. Se dejan drenajes rígidos con aspiración intermitente a nivel laterocervical, retroesofágico y mediastínico (Fig. 5), todos ellos colocados a partir de la incisión cervical. No se 


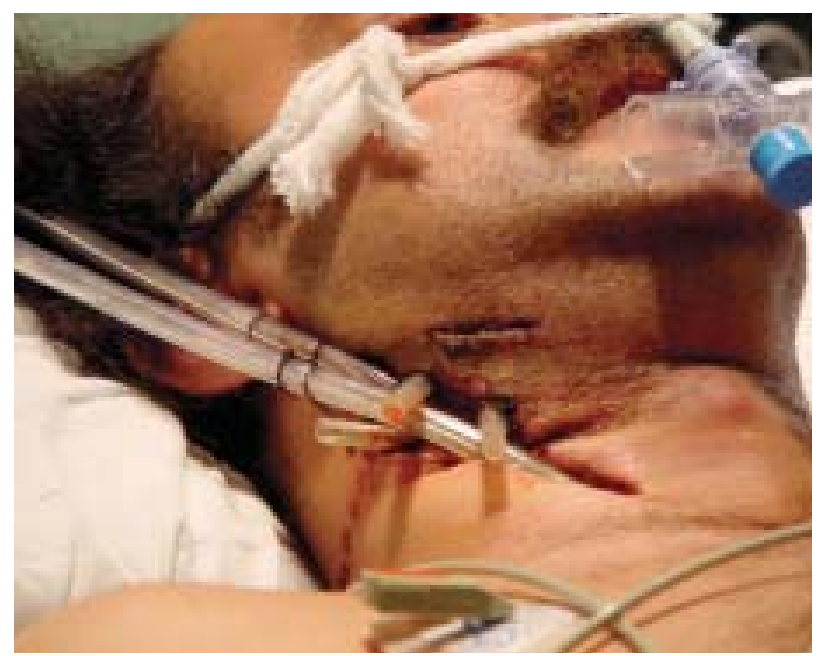

Figura 5. Colocación de drenajes en región submandibular, retroesofágico y hacia espacio mediastínico.

realiza traqueostomía, permaneciendo intubado en la unidad de Cuidados Intensivos.

Durante los siguientes cuatro días se objetiva salida de material purulento a través de los drenajes cervicales y el quinto día, tras realizar bronco-fibroscopia previa a la extubación, se evidencia granuloma traqueal de origen desconocido que hace mayor el riesgo de oclusión de la vía aérea a la extubación, por lo que se decide realizar una traqueostomía reglada (Fig. 6) y cambio de drenajes. Tras los resultados del cultivo del material purulento, donde crecen diferentes familias de Streptococo (anginosus e intermedius), Pseudomona aeruginosa y Prevotella ruminicula, se instaura tratamiento antibiótico en base a los resultados del antibiograma con Piperacilina junto con Tazobactam $4 \mathrm{~g}$ cada 8 horas endovenoso y Ornidazol $1 \mathrm{~g}$ cada 24 horas endovenoso; 48 horas tras la traqueostomía el paciente ingresa en planta desde la UCI. Durante los días posteniores el paciente presenta una correcta evolución clínica (Fig. 7), por lo que tras 22 días de ingreso es dado de alta previa exodoncia de las piezas 47 y 48 en Consultas Externas de nuestro Servicio bajo anestesia loco-regional, retirada de los drenajes y cierre del traqueostoma mediante aproximación de bordes.

\section{Discusión}

La necesidad de realizar una traqueostomía en un mismo acto quirúrgico ante toda infección grave de espacios cervicales es un tema ampliamente discutido en la literatura con autores a favor y en contra, ${ }^{8-}$ 11 debido al riesgo real de diseminación del proceso infeccioso hacia mediastino o incluso hacia la vía aérea con el consiguiente aumento de la morbi-mortalidad. También existe discrepancia en cuanto al momento ideal de realizar la exodoncia de las piezas que se consideran causantes del proceso infeccioso. Por el contrario existe bastante uniformidad en



Figura 6. Realización de traqueostomía reglada.

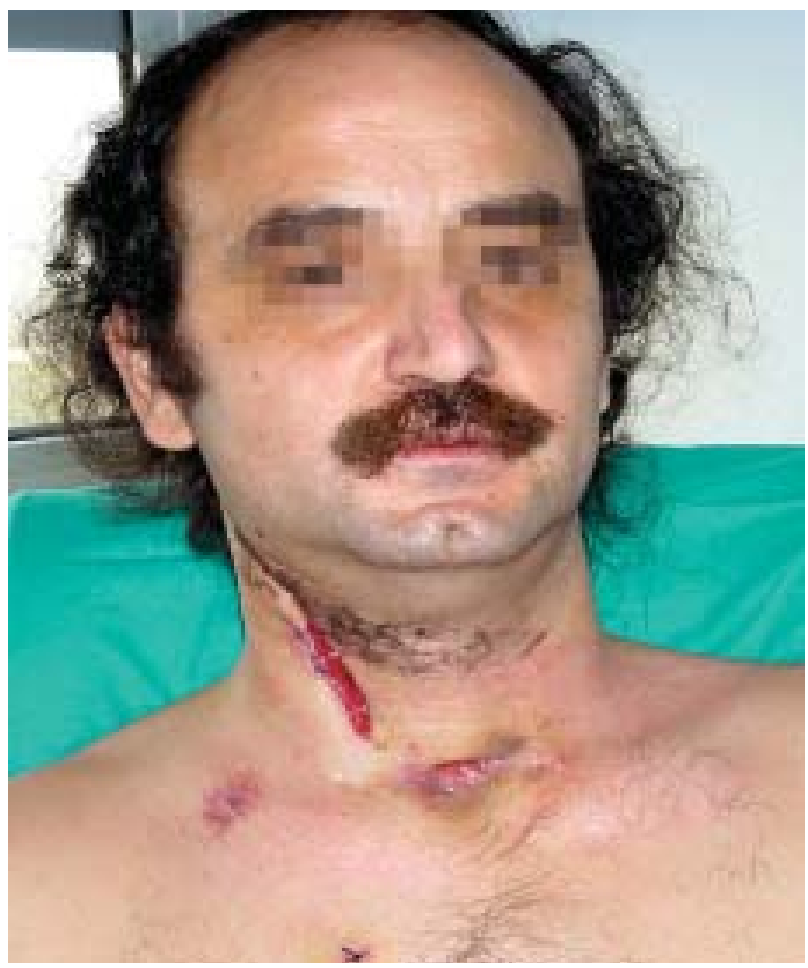

Figura 7. Paciente durante su fase final de hospitalización, en resolución del cuadro infeccioso.

cuanto a la importancia del tiempo desde que el paciente es explorado hasta que se toma la decisión terapéutica, debido al curso fulminante que pueden seguir estos procesos infecciosos y dado que el riesgo de mortalidad puede deberse a compromiso de la vía aérea o por shock séptico. Nosotros estamos a favor de realizar la traqueostomía siempre que la vía aérea sufra un serio compromiso y la intubación no puede realizarse de forma atraumática (mediante fibroscopio) y/o cuando se prevea una larga intubación postoperatoria. 


\section{Conclusiones}

a. Un desbridamiento quirúrgico amplio y precoz es, junto con una antibioterapia endovenosa específica, la base para un correcto tratamiento de esta patología. Es importante, mientras se esperan los resultados del antibiograma, el iniciar una antibioterapia endovenosa de amplio espectro para cubrir tanto gérmenes aerobios como anaerobios.

b. Consideramos que la traqueostomía no debe ser una intervención sistemática en este tipo de pacientes sino más bien una maniobra para mantener una vía aérea permeable en los casos que así se requiera, por el riesgo que conlleva esta técnica quirúrgica en pacientes con infecciones graves en espacios cervicales.

c. En cuanto a la exodoncia de las piezas origen de la infección, creemos necesario el realizarla en un segundo tiempo quirúrgico en aquellos casos en los que se prevea una extracción difícil con amplio fresado óseo para evitar así el niesgo de contacto entre tejidos infectados y aquellos que no están en contacto directo con la infección.

\section{Agradecimientos}

Dr. J. Moya, Médico Adjunto del Servicio de Cirugía Torácica del HUB y Profesor de Anatomía Humana de la Universidad de Barcelona.

\section{Bibliografía}

1. Steiner M, Grau MJ. Odontogenic infection leading to cer- vical emphysema and fatal mediastinitis. J Oral Maxillofac Surg 1982;40:600-4.

2. Tung-Yiu W, Jehn-Shyun H. Cervical necrotizing fascitis of odontogenic origin. A report of 11 cases. J Oral Maxillofac Surg 2000;58:1347-52.

3. McCurdy JA Jr, MacInnis EL, Hays L. Fatal mediastinitis after dental infection. J Oral Surg 1977; 35:726-9.

4. Moriwaki, Yoshihiro, Sugyyama, Mitsugi. Approach for drainage of descending necrotizing mediastinitis on the basis of the extending progression from deep neck infection to mediastinitis. Journal of Trauma Injury, Infection and Critical Care 2002;53:112-6.

5. Weathley MJ, Stirling MC, Kirsch MM. Descending necrotizing mediastinitis: trancervical drainage is not enough. Ann Thoracic Surg 1990;49:780-4.

6. Sakamoto H, Aoki T, Kise Y, Watanabe D. Descending necrotizing mediastinitis due to odontogenic infections. Oral Surg Oral Med Oral Pathol Oral Radiol Endod 2000;89:412-9.

7. Williams AC, Guralnick WC. The Diagnosis and treatment of Ludwig's angina. N Engl J Med 1943; 228:443.

8. Moncada R, Warpeha R, Pickleman J, Spak M. Mediastinitis from odontogenic and deep cervical infections: anathomical pathways of propagation. Chest 1978;73: 497500.

9. Brunelli A, Sabbatini A, Catalini G, Fianchini A. Descending necrotizing mediastinitis. Surgical drainage and tracheostomy. Arch Otolaring Head Neck Surg 1996;122: 1326-9.

10. Allen D, Loughnan TE, Ord RA. A reevaluation of the role of tracheotomy in Ludwig's angina. J Oral Maxillofac Surg 1985;43:436-9.

11. Bounds GA. Subphrenic and mediastinal abscess formation. A complication of Ludwig's angina. Br J Oral Maxillofac Surg 1985;23:313-21. 